\title{
Escala de Necessidade de Identificação: Propriedades Psicométricas em Contexto Brasileiro ${ }^{1}$
}

\author{
Anderson Mesquita do Nascimento², Valdiney Veloso Gouveia \\ Universidade Federal da Paraíba, João Pessoa-PB, Brasil \\ Ana Karla Silva Soares \\ Universidade Federal do Mato Grosso do Sul, Campo Grande-MS, Brasil \\ Jéssica Bruna Santana Silva, Alex Sandro de Moura Grangeiro \\ Universidade Federal da Paraíba, João Pessoa-PB, Brasil
}

\section{RESUMO}

A necessidade de identificação com grupos reflete uma tendência individual a se identificar com grupos ou metas sociais. Esta pesquisa procura adaptar a Escala de Necessidade de Identificação (ENI) para o contexto brasileiro, contando com dois estudos sobre suas evidências de validade fatorial e consistência interna. O Estudo 1 incluiu 244 participantes (idade média=21,9; 66,3\% mulheres), tendo os resultados sugerido uma estrutura de dois fatores: Autodefinição e Pertencimento. O Estudo 2 reuniu 246 participantes (idade média $=21,3 ; 68,7 \%$ mulheres), confirmando essa estrutura de dois fatores, reunindo indicadores de ajuste satisfatórios (e.g., $\mathrm{CFI}=0,96, \mathrm{TLI}=0,94)$. Em ambos os estudos, os fatores demonstraram índices de fidedignidade adequados. Concluiu-se que a versão brasileira da ENI reuniu evidências psicométricas adequadas, apoiando seu uso no Brasil para avaliar a tendência individual a se definir como membro de um grupo.

Palavras-chave: necessidade, identificação, validade, fidedignidade, escala.

\section{ABSTRACT - Identification need scale: psychometric properties in the Brazilian context}

The need for group identification reflects an individual tendency to identify with social groups or goals. This research seeks to adapt the Identification Need Scale (INS) to the Brazilian context, counting on two studies on its factorial validity evidence and internal consistency. Study 1 included 244 participants (mean age $=21.9,66.3 \%$ female), and the results suggested a two-factor structure: selfdefinition and belonging. Study 2 included 246 participants (mean age $=21.3,68.7 \%$ female), confirming this two-factor structure, with satisfactory adjustment indicators (e.g., $\mathrm{CFI}=0.96, \mathrm{TLI}=0.94)$. In both studies the factors demonstrated adequate reliability indices. It was concluded that the Brazilian version of INS gathered adequate psychometric evidence, supporting its use in Brazil to evaluate the individual tendency to define itself as a member of a group.

Keywords: need; identification; validity; reliability; scale.

\section{RESUMEN - Escala de Necesidad de Identificación: Propiedades Psicométricas en el Contexto Brasileño}

La necesidad de identificación con grupos, refleja una tendencia individual a identificarse con grupos o metas sociales. Esta investigación busca adaptar la Escala de Necesidad de Identificación (ENI) en el contexto brasileño, contando con dos estudios sobre evidencias de validez factorial y consistencia interna. El Estudio 1 incluyó 244 participantes (edad promedio=21,9 anõs; 66,3\% mujeres) y los resultados mostraron una estructura de dos factores: autodefinición y pertenencia. El Estudio 2 incluyó 246 participantes (edad promedio=21,3 anõs; $68,7 \%$ mujeres) confirmando la estructura de dos factores, reuniendo los indicadores de ajuste satisfactorios (e.g., CFI=0,96, TLI=0,94). En ambos estudios, los factores mostraron índices adecuados de confiabilidad. La versión brasileña de ENI reunió evidencias psicométricas adecuadas, apoyando su uso en Brasil para evaluar la tendencia individual a definirse como miembro de un grupo.

Palabras clave: necesidad; identificación; validez; confiabilidad; escala.

Em todas as sociedades, os indivíduos mantêm relações interpessoais em uma rede de grupos, que são importantes por proporcionarem o apoio social e um modelo cultural que guia o comportamento individual (Brewer \& Yuki, 2007). Segundo Torres e Neiva (2011), os grupos sociais podem ser definidos como duas ou mais pessoas que interagem entre si e são interdependentes com relação a suas necessidades e objetivos. Pode decorrer desse entendimento que o grupo consiste em uma unidade social que possui atributos específicos, a saber: filiação, interação entre seus integrantes, objetivos e normas compartilhadas (Michener, DeLamater, \& Myers, 2005).

${ }^{1}$ Os autores agradecem à CAPES pelo financiamento através de bolsa de estudos ao primeiro e quinto autores, e ao CNPq pelo financiamento através de bolsa de produtividade ao segundo autor.

Endereço para correspondência: E-mail: andermnascimento@gmail.com 
Desse modo, fazer parte de um grupo demanda compartilhar algum tipo de envolvimento com seus membros, formando-se por meio dessa relação o que se denomina como identidade social (Torres \& Neiva, 2011).

O estudo do processo de identificação com grupos se baseia nos pressupostos da Teoria da Identidade Social (Tajfel \& Turner, 1979) e Teoria da Autocategorização (Turner, 1982). A primeira, segundo Tajfel (1978), diz respeito ao autoconceito que deriva do pertencimento a um ou mais grupos e do valor e significado emocional atribuídos a essa filiação. No caso da Teoria da Autocategorização, têm como suporte a categorização e a comparação social. Segundo a primeira premissa, os indivíduos organizam sua compreensão do mundo social com base nas distinções entre categorias sociais, o que minimiza as diferenças percebidas no endogrupo e acentua aquelas entre endogrupo-exogrupo; a segunda premissa sugere que os membros do endogrupo são percebidos como mais similares ao "eu" do que aqueles do exogrupo (Tajfel, Flament, Billig, \& Bundy, 1971).

A partir desses pressupostos teóricos, observa-se que a identidade social tem sido investigada com ênfase no caráter social da identificação grupal, compreendendo-a como dependente do contexto social no qual o indivíduo está inserido. Contudo, Mayhew, Gardner e Ashkanasy (2010) argumentam que esse foco contextual não é capaz de explicar os diferentes níveis de identificação que os indivíduos apresentam com os grupos, ainda que estes se encontrem em contexto similar. Desse modo, esses autores atribuem tal fato a influência de diferenças individuais no processo de identificação social, buscando explorar o papel do indivíduo nesse processo ao propor que as pessoas variam na propensão a se identificarem com grupos ou metas sociais, tendo lugar então o construto denominado como Necessidade de Identificação (NI).

A necessidade de identificação pode ser conceituada como uma tendência individual a se definir como membro de um grupo. Esta não permanece constante em todas as situações, pois, para que o indivíduo possa se identificar com dado grupo social, faz-se necessário inicialmente que tal grupo seja posto em evidência (Haslam, 2001). Nessa perspectiva, os indivíduos são suscetíveis a se identificarem fortemente com um grupo quando eles têm alta NI, porém isso ocorre apenas quando o contexto enfatiza esse grupo, isto é, quando reforça o pertencimento ao invés da individualidade (Mayhew et al., 2010).

A NI envolve ainda o equilíbrio entre pertencimento e distinção com o grupo. De acordo com Brewer (1991), indivíduos visam a um estado de distintividade ótima equilibrando um anseio por participação em categorias sociais com uma necessidade por individualidade baseada na diferenciação entre o eu e os outros. Entretanto, segundo esse autor, o nível de NI não é constante entre os indivíduos, visto que aqueles que apresentam alta NI se mostram como favoráveis ao pertencimento a grupos, enquanto aqueles com baixa NI são favoráveis à distinção em detrimento da pertença a grupos sociais.

Outro aspecto que está teoricamente relacionado com a NI é o autoconceito. A Teoria da Identidade Social parte da concepção de que o autoconceito deriva do pertencimento a um ou mais grupos e do valor e significado emocional atribuído a essa filiação (Tajfel, 1978). Os indivíduos diferem em relação ao autoconceito; para algumas pessoas, envolve uma interpretação independente do eu, que é visto como autônomo, enquanto que outras possuem um autoconceito interdependente, ou seja, são mais conectadas com outros membros grupais, de modo que essas relações os definem como indivíduo (Markus \& Kitayama, 1991). A partir dessa perspectiva teórica, a variação da NI pode levar a diferenças na formação do autoconceito, pois indivíduos com alta NI seriam mais prováveis a constituir o autoconceito como interdependente e conectado com os membros do grupo social; contrariamente, aqueles com baixa NI poderiam apresentar uma interpretação mais independente do eu (Mayhew et al., 2010).

A identificação com grupos tem importantes implicações na vida cotidiana. Por exemplo, Greenaway et al. (2015) observaram que a identificação com grupos eleva o controle pessoal percebido, com consequências positivas na saúde mental e bem-estar. Além disso, a identificação com grupos também pode proteger contra estados mentais não saudáveis, tais como a ansiedade e depressão (Cruwys, South, Greenaway, \& Haslam, 2015). Considerando a relevância da NI para a saúde mental dos indivíduos e sua importância para a compreensão do processo de identificação grupal, Mayhew et al. (2010), após verificar a inexistência de medidas prévias que trate de grupos em geral, decidiram elaborar a Escala de Necessidade de Identificação (ENI).

Essa escala buscou refletir uma tendência de identificação generalizada para todos os contextos sociais. Ressalta-se, nesse ponto, que já existe, em contexto brasileiro, uma medida sobre identificação com grupos (Pimentel, Gouveia, \& Fonseca, 2005), porém é mais restrita, tendo em conta apenas grupos alternativos (e. g., funkeiros, skatistas, metaleiros); essa é uma proposta diferente da ENI, que procura avaliar uma tendência geral de identificação com grupos sociais. Desse modo, a adaptação da ENI ao contexto brasileiro traz a vantagem de contar com uma medida que avalie a identificação com grupos, independentemente do grupo avaliado, não sendo necessário adaptar uma nova medida toda vez que o grupo posto em evidência seja modificado.

A Escala de Necessidade de Identificação (ENI) foi desenvolvida em contexto australiano por Mayhew et al. (2010). A fim de contar com itens que representassem adequadamente o construto de interesse, Mayhew et al. (2010) submeteram o conjunto inicial de 37 itens (sete de uma medida de identificação organizacional e 30 elaborados a partir da literatura) à apreciação de oito juízes, pesquisadores em psicologia social/organizacional 
(precisão interjuízes de 0,70$)$. Nesse momento, foi observada a necessidade de exclusão de sete itens. Após essa etapa, Mayhew et al. (2010) avaliaram, em quatro estudos independentes, as evidências de validade e precisão (consistência interna e estabilidade temporal) da versão preliminar da escala, composta por 30 itens. Nos Estudos 1 e 2, os autores originais do instrumento procuraram conhecer a estrutura fatorial da ENI por meio das análises fatoriais exploratórias e confirmatórias. Dos 30 itens iniciais, restaram apenas 11, distribuído em dois fatores (autodefinição e pertencimento). Essa estrutura foi replicada em estudos posteriores conduzidas pelos mesmos autores (Estudo 3 e 4), os quais, além de atestarem a adequabilidade da solução fatorial proposta, demonstraram a boa estabilidade temporal ( $r>0,40$; teste-reteste) e a aceitável consistência interna $(\alpha>0,70)$ da medida.

Em resumo, considerando a relevância do construto necessidade de identificação com grupos em diversas áreas da Psicologia (e. g., clínica, social, organizacional, escolar) e seu impacto na saúde mental e bem-estar (Cruwys et al., 2015; Greenaway et al., 2015), inexistindo na realidade brasileira uma medida com o propósito de avaliar essa tendência geral ou predisposição do indivíduo para se identificar com tais grupos (Pimentel et al., 2005), decidiu-se adaptar a ENI para a realidade brasileira. Nesse sentido, realizaram-se dois estudos para checar sua estrutura fatorial e consistência interna, que são descritos a continuação.

\section{Estudo 1. Conhecendo a Estrutura Fatorial da ENI no Contexto Brasileiro}

Em razão de não terem sido encontrados achados sobre a adaptação da Escala de Necessidade de Identificação (ENI) na realidade brasileira, decidiu-se checar inicialmente sua estrutura fatorial e a consistência interna do(s) fator(es) resultante(s).

\section{Método}

\section{Participantes}

A amostra foi escolhida por critério de conveniência (seleção não probabilística), contando com 244 estudantes universitários com idades entre 16 e 57 anos $(M=21,9$, $\mathrm{DP}=6,27)$, a maioria do sexo feminino $(66,3 \%)$ e solteira $(85,6 \%)$ de uma instituição de ensino superior do estado da Paraíba e distribuídos entre os cursos de Psicologia (53,3\%), Direito (18,9\%), Engenharia Civil (7,8\%), Fisioterapia (6,1\%), Enfermagem (5,7\%), Nutrição (3,7\%), Farmácia (3,7\%) e Química Industrial (0,8\%).

\section{Instrumentos}

Os participantes receberam uma versão lápis-papel da Escala de Necessidade de Identificação (ENI), desenvolvida originalmente em língua inglesa por Mayhew et al. (2010), constando de onze itens (e. g., "Tenho muito em comum com os outros membros dos grupos que participo; Fazer parte de grupos me dá uma identidade"). A respostas para esses itens foram dadas em escala tipo Likert, de 5 pontos, variando de 1 (Discordo totalmente) a 5 (Concordo totalmente). Além desse instrumento, todos responderam a um questionário com perguntas de cunho demográfico (idade, sexo e estado civil).

Foi utilizado o método back-translation para elaboração da versão em português da ENI. Assim, inicialmente a medida foi traduzida do inglês para o português por um colaborador, sendo essa versão traduzida novamente para o inglês por uma segunda colaboradora. A partir das traduções, procedeu-se a comparação das versões, contando com a participação de um terceiro colaborador. Todos os colaboradores eram bilíngues. Em seguida, observou-se a presença de equivalência semântica das medidas e nenhuma modificação substancial foi necessária, mantendo-se todos os itens da versão original em inglês.

\section{Procedimento}

Após autorização dos professores das disciplinas, solicitou-se que os estudantes presentes respondessem voluntariamente à ENI, que era administrada no início ou término das aulas, contando com a colaboração de dois pesquisadores aptos a proceder à aplicação. Os estudantes responderam individualmente à medida, embora o tenham feito em ambiente coletivo de sala de aula. O tempo médio para finalizar a participação na pesquisa foi de 5 minutos. Todos foram informados sobre a confidencialidade das respostas, esclarecendo que também poderiam desistir da pesquisa a qualquer momento sem ônus. Esta pesquisa teve parecer favorável do Comitê de Ética do Centro de Ciências Médicas, da Universidade Federal da Paraíba ( $\mathrm{n}^{\circ}$ 1.176.964), conforme recomenda a Resolução no 466/12, do Conselho Nacional de Saúde, bem como seguiu as recomendaçãoes da Declaração de Helsinki (1975, revisada em 1983) concernente às pesquisas com seres humanos.

\section{Análise dos Dados}

Para conhecer a dimensionalidade da ENI, realizou-se uma análise fatorial exploratória Minimum Rank Factor Analysis (Shapiro \& ten Berge, 2002), empregando rotação oblíqua Promin (Lorenzo-Seva, 1999), que foi conduzida com o Factor 10.3 (Lorenzo-Seva \& Ferrando, 2006). Como método de retenção fatorial, utilizou-se o Hull-Comparative Fit Index (Lorenzo-Seva, Timmerman, \& Kiers, 2011), que visa identificar qual estrutura fatorial apresenta melhor ajuste para os dados observados e seus respectivos graus de liberdade. Foram utilizadas as matrizes de correlação policóricas para reter a estrutura fatorial da medida (Holgado-Tello, Chacón-Moscoso, BarberoGarcía, \& Vila-Abad, 2010). Além disso, foram estimados os índices de fidedignidade dos fatores de Mislevy (Mislevy \& Bock, 1990), considerando valores acima de 0,70 como satisfatórios (Kline, 2000). Esse índice pode 
ser utilizado em alternativa ao alfa de Cronbach, visto que este último se baseia em correlações de Pearson, não sendo adequado para dados categóricos-ordenados. Além disso, observou-se violação dos critérios de normalidade univariada e multivariada do conjunto dos dados $($ Mardia $=153,15 ; p<0,05)$, endossando a utilização dessas análises.

\section{Resultados}

O Kaiser-Meyer-Olkin $(\mathrm{KMO}=0,85)$ e o Teste de Esfericidade de Bartlett $\left[\chi^{2}(\mathrm{df})=972,20, p<0,001\right]$ demonstraram a adequação da matriz de correlações policóricas para a realização da análise fatorial exploratória. Uma análise preliminar revelou a presença de dois fatores com valores próprios acima de 1, mesma estrutura indicada posteriormente pelo critério Hull-CFI $(\mathrm{CFI}=0,93, g l=140)$, o que pode ser observado pela melhor proporção entre o índice de ajuste e os graus de liberdade desse modelo em relação aos demais modelos possíveis, como descrito na Tabela 1.

Tabela 1

Índices de Ajuste Apresentados por Diferentes Soluções Fatoriais segundo o Critério HULL-CFI

\begin{tabular}{cccc}
\hline $\begin{array}{c}\text { Número de } \\
\text { Fatores }\end{array}$ & CFI & gl & $\begin{array}{c}\text { Valores no } \\
\text { Scree-test }\end{array}$ \\
\hline 0 & 0,00 & 11 & 0,00 \\
1 & 0,73 & 22 & 3,42 \\
2 & 0,93 & 32 & $4,75^{*}$ \\
3 & 0,96 & 41 & 0,00 \\
\hline
\end{tabular}

Nota. *Número de fatores recomendado pelo critério HULL-CFI
Os dois fatores encontrados explicaram conjuntamente $54,7 \%$ da variância total. Conforme a Tabela 2 , o primeiro fator reuniu quatro itens com cargas fatoriais variando de 0,74 (Item 10. Sem os grupos que faço parte, eu me sentiria incompleto) a 0,82 (Item 11. Meus grupos ilustram quem eu sou). Ele foi denominado como "autodefinição", apresentando índice de fidedignidade de 0,87 . O segundo fator também reuniu quatro itens com cargas fatoriais variando de 0,61 (Item 1. Tenho muito em comum com os outros membros dos grupos que participo) a 0,78 (Item 5. Sinto-me confortável com outras pessoas que sabem que participo de grupos). Esse fator foi nomeado como "pertencimento", apresentando índice de fidedignidade de 0,83 . Foi utilizado como critério para definir a pertença do item ao fator, saturação igual ou superior a 0,30 , sendo eliminados os itens com saturação inferior a recomendada ou com saturações superiores ao critério adotado em ambos os fatores. Assim, ressalta-se que os itens 6 e 7 apresentaram cargas fatoriais acima de 0,30 nos dois fatores, enquanto que o item 3 não alcançou carga fatorial figurar em qualquer fator, sendo eles excluídos de análises posteriores.

\section{Estudo 2. Comprovação da Estrutura de Dois Fatores da ENI}

Esse estudo não é exatamente uma replicação do anterior, mas compreende um esforço por ampliar evidências psicométricas da ENI, reunindo achados sobre sua validade fatorial e consistência interna. Essencialmente, foca-se em procedimentos confirmatórios, avaliando se a solução de dois fatores é a mais adequada para representar os itens de necessidade de identificação.

Tabela 2

Estrutura Fatorial da Escala de Necessidade de Identificação $(n=241)$

\begin{tabular}{|c|c|c|}
\hline Item & $\mathrm{I}^{*}$ & $\mathrm{II}^{* *}$ \\
\hline 11. Meus grupos ilustram quem eu sou. & 0,82 & $-0,05$ \\
\hline 09. Meu entendimento de quem eu sou vem dos grupos que faço parte. & 0,80 & $-0,07$ \\
\hline 08. Fazer parte de grupos me dá uma identidade. & 0,76 & 0,05 \\
\hline 10. Sem os grupos que faço parte, eu me sentiria incompleto. & 0,74 & $-0,15$ \\
\hline 05. Sinto-me confortável com outras pessoas que sabem que participo de grupos. & $-0,07$ & 0,78 \\
\hline 04. Prefiro dizer "nós" a "eles" quando falo dos grupos que faço parte. & $-0,27$ & 0,75 \\
\hline 02. Gosto de fazer parte dos grupos que participo. & $-0,12$ & 0,75 \\
\hline 01. Tenho muito em comum com os outros membros dos grupos que participo. & 0,02 & 0,61 \\
\hline 07. Ser um membro de grupos fornece-me com um forte senso de quem eu sou & 0,41 & 0,44 \\
\hline 06. Quando eu penso sobre mim mesmo, eu penso sobre os grupos dos quais eu faço parte. & 0,32 & 0,47 \\
\hline $\begin{array}{l}\text { 03. Não importa qual grupo eu pertenço, eu gostaria de pensar em mim mesmo } \\
\text { como representando o que esse grupo representa. }\end{array}$ & 0,06 & 0,28 \\
\hline Número de itens & 4 & 4 \\
\hline Variância explicada & $39,16 \%$ & $15,58 \%$ \\
\hline Índice de fidedignidade & 0,87 & 0,83 \\
\hline
\end{tabular}

Nota. *Fator I: Autodefinição; **Fator II: Pertencimento 


\section{Método}

\section{Participantes}

Contou-se com uma amostra de conveniência (não probabilística) formada por 246 estudantes universitários de uma instituição de ensino superior de João Pessoa, PB. Estes tinham idades variando de 16 a 48 anos $(\mathrm{M}=21,3, \mathrm{DP}=5,78)$, sendo a maioria do sexo feminino $(68,7 \%)$, solteira $(87,1 \%)$ e distribuídos entre os cursos de Psicologia (46\%), Direito (25\%), Enfermagem (9\%), Engenharia Civil (7\%), Fisioterapia (5\%), Nutrição (5\%), Farmácia (1,5\%) e Química Industrial (1,5\%).

\section{Instrumentos e Procedimento}

Os participantes responderam os mesmos instrumentos do Estudo 1, reunindo a Escala de Necessidade de Identificação e perguntas demográficas. Seguiram-se as mesmas determinações éticas previamente citadas, assegurando o anonimato da participação e confidencialidade das respostas, explicitando que os respondentes poderiam deixar o estudo se o desejassem sem que isso implicasse qualquer ônus. Os participantes responderam os mesmos instrumentos do Estudo 1, reunindo a Escala de Necessidade de Identificação e perguntas demográficas. Seguiram-se as mesmas determinações éticas previamente citadas, assegurando o anonimato da participação e confidencialidade das respostas, explicitando que os respondentes poderiam deixar o estudo se o desejassem sem que isso implicasse qualquer ônus. Cabe ressaltar que a coleta para ambos os estudos foi realizada de uma única vez e a amostra foi dividida em duas partes para proceder às análises.

\section{Análise dos Dados}

Por meio de análise fatorial confirmatória, testaram-se os modelos de dois fatores, como originalmente proposto e observado no Estudo 1, e um modelo unifatorial alternativo, reunindo todos os itens, os quais foram comparados diretamente (Kline, 2011). Utilizou-se como entrada a matriz de correlação policórica, adotando-se o estimador Weighted Least Squares Means and Variance adjusted (WLSMV; Muthén, Du Toit, \& Spisic, 1997). Essas análises foram realizadas com o Mplus 6.12 (Muthén \& Muthén, 2010). A comprovação do ajuste dos modelos aos dados empíricos considerou múltiplos indicadores: o Comparative Fit Index (CFI) e Tukey-Lewis Index (TLI), demandando-se valores de 0,90 ou superiores como indicação de ajuste adequado; o Root Mean Square Error of Approximation (RMSEA), cujo valor deve ser menor do que 0,05 , admitindo-se até 0,10 como limite superior de seu intervalor de confiança (IC90\%); e o $\chi^{2}$ (gl) ao nível de signicância de 0,05 . A consistência interna dos fatores foi comprovada com o índice ômega de Mcdonald ( $\omega)$, obtido por meio do programa $\mathrm{R}$ ( $R$ Development Core Team, 2011), utilizando o pacote semTools (Pornprasertmanit, Miller, Schoemann, \& Rosseel, 2015). Nesse caso, o ponto de corte utilizado foi de 0,70 (Kline, 2000).

\section{Resultados}

Embora não tenha sido perfeito, o modelo de dois fatores $[\mathrm{CFI}=0,96, \mathrm{TLI}=0,94, \mathrm{RMSEA}=0,08$ $(\mathrm{IC} 90 \%=0,06-0,11)$ e $\left.\chi^{2}(19)=53,37, p<0,001\right]$ apresentou melhores indicadores de ajuste do que o unifatorial $[\mathrm{CFI}=0,88, \mathrm{TLI}=0,83, \mathrm{RMSEA}=0,15 \quad(\mathrm{IC} 90 \%=0,12-$ $0,17)$ e $\left.\chi^{2}(20)=128,77, p<0,001\right]$. O primeiro modelo também apresentou um incremento no ajuste em termos da dimensionalidade da ENI em relação ao segundo, produzindo menos resíduos entre o modelo predito e a matriz empírica $\left[\Delta \chi^{2}(1)=42,51, p<0,001\right]$. $\mathrm{Na}$ Figura 1, o modelo de dois fatores é representado, incluindo as saturações dos itens e a correlação entre os fatores.

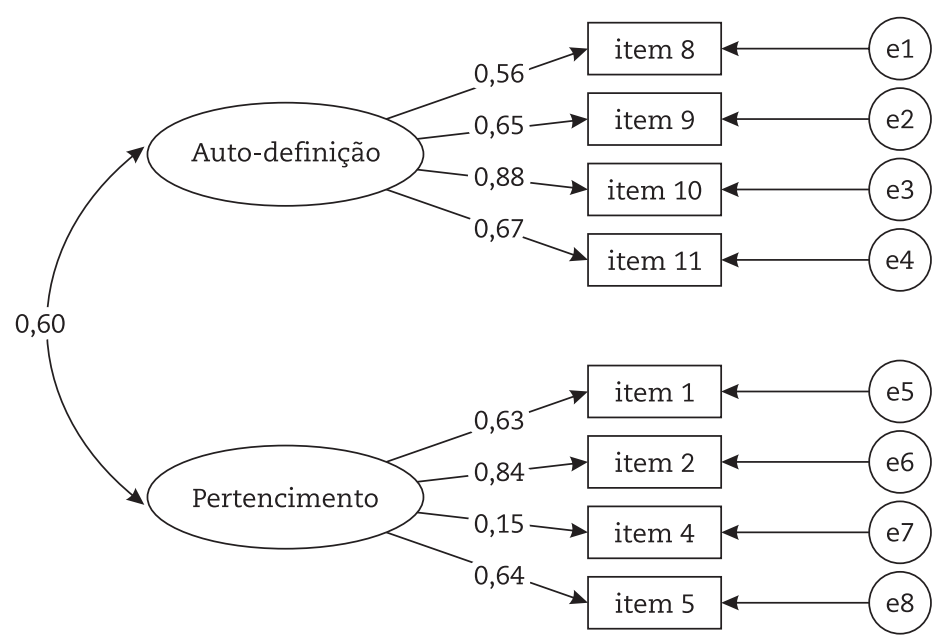

Figura 1. Estrutura fatorial da escala de Necessidade de Identificação $(n=246)$ 
Destaca-se que as saturações foram todas positivas e diferentes de zero $(\lambda \neq 0 ; p<0,001)$. No caso do fator autodefinição, elas variaram de 0,56 (Item 4 . Fazer parte de grupos me dá uma identidade) a 0,88 (Item 10. Sem os grupos que faço parte, eu me sentiria incompleto), apresentando ômega $(\omega)$ de 0,65 . Por sua vez, no fator Pertencimento as saturações variaram de 0,15 (Item 4. Prefiro dizer "nós" a "eles" quando falo dos grupos que faço parte) a 0,84 (Item 2. Gosto de fazer parte dos grupos que participo), resultando em $\omega$ de 0,83 .

\section{Discussão Geral}

A necessidade de identificação (NI) pode ser caracterizada como uma tendência individual a se autodefinir como membro de um grupo, visto que as pessoas variam na propensão a se identificar com grupos ou metas sociais, ainda que estes se encontrem em contexto similar (Haslam, 2001). Uma vez que a NI pode auxiliar no entendimento da influência das diferenças individuais na identificação grupal, pareceu relevante adaptar ao contexto brasileiro a Escala de Necessidade de Identificação (ENI; Mayhew et al., 2010), reunindo evidências de sua validade fatorial e consistência interna.

A análise fatorial exploratória Minimum Rank Factor Analysis (Shapiro \& ten Berge, 2002), empregando o método de retenção Hull-CFI, identificou uma estrutura de dois fatores equivalente àquela do estudo original dessa medida (Mayhew et al, 2010). Contudo, tais fatores retiveram menos itens; concretamente, o item 3 não alcançou saturação mínima em qualquer dos fatores e os itens 6 e 7 saturaram acima do ponto de corte em ambos os fatores. Semanticamente, esses itens misturam elementos de autodefinição (e. g., fornecer um senso de quem é, pensar sobre si mesmo) com aqueles de pertencimento grupal (e. g., ser o membro de um grupo, pensar sobre os grupos do qual faz parte). Optou-se por não fazer modificações prévias nesses itens antes da aplicação dos questionários devido à possibilidade de os itens se reunirem em um fator geral de NI. Como isso não ocorreu, preferiu-se excluir esses itens do conjunto para não causar problemas na interpretação dos fatores. Os demais itens saturaram em seus fatores originais, que apresentaram consistência interna compatível com estudos prévios (Mayhew et al., 2010) e consoantes com o recomendado (Kline, 2000).

No estudo 2, procedeu-se com análises de cunho confirmatório, para verificar se o modelo com dois fatores apresenta bom ajuste aos dados de uma nova amostra, contrastando-o com uma solução mais simples, isto é, unifatorial. Os resultados apoiaram a adequação da estrutura constituída por dois fatores (Autodefinição e Pertencimento), reunindo oito itens distribuídos equitativamente, com indicadores de ajuste satisfatórios (Muthén, Du Toit, \& Spisic, 1997; Tabachnick \& Fidell, 2013). Verificou-se, ainda, que os fatores apresentaram coeficientes de consistência interna próximos ou superiores aos sugeridos na literatura; o fator Autodefinição não alcançou 0,70 , mas há que se considerar a natureza psicológica desse construto (Kline, 2000), que não compreende um traço de personalidade ou uma habilidade cognitiva, que se esperaria mais homogêneo ou consistente (Gouveia, Santos, \& Milfont, 2009). Portanto, entende-se que também esse fator reuniu evidência de consistência interna.

No que diz respeito aos achados principais, corroborou-se a estrutura de dois fatores dessa medida, que claramente abarca os fatores de Autodefinição e Pertencimento (Mayhew et al., 2010). Para indivíduos com pontuação alta em Autodefinição a interação grupal fornece uma forte identidade e senso de si, o que encontra respaldo quando considerada a relação teórica entre a NI e a Teoria da Identidade Social (Tajfel \& Turner, 1979). Vale ressaltar que essa parte da concepção de que o autoconceito deriva do pertencimento a um ou mais grupos e do valor e significado emocional atribuído a esta filiação (Tajfel, 1978). Nesse caso, quanto mais o indivíduo se autodefine como membro de um grupo maior será a contribuição da pertença a esse grupo para a formação do autoconceito desse indivíduo. Por outro lado, o fator Pertencimento corresponde ao desejo de pertencer e compartilhar algum tipo de envolvimento com o grupo, já que este consiste em uma unidade social que possui como atributos a afiliação, interação entre os integrantes, normas e objetivos compartilhados (Michener et al., 2005). Em indivíduos com pontuações altas nesse fator a associação a um grupo proporciona uma sensação de afinidade com base na semelhança, no prazer e na unidade (Mayhew et al., 2010).

Foi observado que os dois fatores apresentaram relação positiva e moderada entre si. Estima-se que essa relação ocorra devido à dependência existente entre esses dois processos para gerar a necessidade de identificação. A autodefinição oferece ao indivíduo o desejo ou propensão a definir a si mesmo em termos do pertencimento a um grupo (Mayhew et al., 2010), no entanto isso não seria suficiente para gerar a necessidade de identificação, visto que também se faz necessário que a adesão ao grupo propicie um aumento da autoestima, reforçando o desejo de pertencer a esse grupo. Considera-se que, apesar dos fatores serem distintos, eles são interdependentes entre si. Portanto, é possível pensar que indivíduos com baixa NI não sejam tão dependentes da participação em grupos sociais para reforçar sua autoestima, valorizando sua distinção em relação aos demais, enquanto que indivíduos com alta NI sejam mais dependentes da participação em grupos para formar uma definição de si mesmos, utilizando-se deles para elevar sua autoestima e formar seu autoconceito.

Em relação ao aspecto estrutural da escala, foram encontradas semelhanças entre as versões observadas no estudo original de desenvolvimento da escala (Mayhew 
et al., 2010) e na presente pesquisa. Tais semelhanças reforçam que a necessidade de identificação é uma tendência individual organizada em dois fatores que, apesar de relacionados, são independentes entre si. Entretanto, também foram verificadas diferenças importantes no conteúdo dos fatores. Especificamente, observou-se menor número de itens retidos por fator decorrente da exclusão de itens que apresentaram saturações nos dois fatores, provavelmente revelando semânticas ou significados similares na cultura brasileira.

É possível pensar em aspectos gerais que tenham ocasionado essas diferenças, sobretudo baseado na diferença entre os contextos culturais onde os estudos foram realizados. No Brasil, predominam elementos de cultura coletivista, onde os indivíduos são concebidos como unidos em uma rede de inter-relações e as instituições são percebidas como uma extensão da família (Gouveia \& Clemente, 2000); isso é diferente do que ocorre na Austrália, onde predominam elementos de cultura individualista (Hofstede, 1984). Além disso, a própria cultura pode funcionar como um grupo que orienta os indivíduos, na qual indivíduos que se identificam fortemente com sua cultura são motivados a agir em concordância com as características normativas desse grupo (Chang, Jetten, Cruwys, \& Haslam, 2017). Portanto, presume-se que, embora a necessidade de identificação compreenda uma tendência individual a se autodefinir como membro de um grupo, fatores contextuais podem influenciar em como os indivíduos definem um grupo e sua forma de participar dele.

Os achados das análises fatoriais exploratória e confirmatória apoiaram essa estrutura de dois fatores. Neste último caso, os indicadores de ajuste foram satisfatórios, geralmente acima dos valores recomendados na literatura (Tabachnick \& Fidell, 2013), tendo sido claramente superiores para a solução de dois fatores quando comparada com a unifatorial. Os dois fatores resultantes, isto é, Autodefinição e Pertencimento, também reuniram evidências de consistência interna, cujos coeficientes foram consoantes com aqueles relatados por Mayhew et al. (2010), que os avaliaram em múltiplos grupos na Austrália. De fato, o menor coeficiente se aproximou ao que tem sido definido como critério mais flexível para checar este parâmetro psicológico (Kline, 2000).

Mesmo que em contexto brasileiro já tenha sido elaborada medida que avalia a identificação grupal (Pimentel et al., 2005), esta é voltada para grupos alternativos específicos. Diferentemente, a ENI se propõe a fazer uma avaliação mais global da identificação com grupos, que é concebida como uma necessidade inerente ao indivíduo. Nesse sentido, é possível que essa medida logre predizer a identificação com grupos variados, incluindo aqueles avaliados no estudo de Pimentel et al. (2005), já que se estima que os indivíduos são mais propensos a se identificarem com um grupo quando eles têm uma NI alta. Porém, vale lembrar, isso somente ocorre quando o contexto enfatiza determinado grupo, explicando uma identificação mais elevada com alguns grupos em detrimento de outros (Mayhew et al., 2010).

Ainda que os objetivos dos estudos tenham sido alcançados, não obsta pensar em suas limitações potenciais. É inevitável reconhecer que as amostras consideradas foram de conveniência, compostas por estudantes universitários, o que reduz o poder de generalização dos achados. Além disso, utilizaram-se estritamente medidas de autorrelato (lápis e papel), que são passíveis de falseamento de respostas, seja por diferenciá-las do real (Kohlsdorf \& Costa, 2009) ou em virtude da desejabilidade social, que pode ocorrer no estudo da necessidade de identificação, visto que, em cultura coletivista como o Brasil, as pessoas buscam se autodefinir como membro de um grupo.

Por fim, entende-se que pesquisas futuras deverão ser realizadas. Uma alternativa seria contemplar estratégias alternativas de medição, a exemplo daquelas de associações implícitas (Gouveia, Athayde, Mendes, \& Freire, 2012). Além disso, também seria pertinente avaliar a necessidade de identificação em amostras diversificadas, incluindo participantes da população geral; checar na presente cultura a estabilidade temporal dessa medida (teste-reteste); e conhecer a associação da NI com outros construtos e condutas, a exemplo de intenção de constituir família, valores humanos, apego ao lugar e altruísmo. Para além do escopo de uma única cultura, estima-se como necessário conhecer a invariância fatorial dessa medida, bem como verificar empiricamente em que medida suas pontuações estão correlacionadas com as orientações individualista e coletivista, quer a nível individual (Gouveia \& Clemente, 2002) ou cultural (Hofstede, 1984), ou com as autoimagens independente e interdependente (Markus \& Kitayama, 1991).

\section{Referências}

Brewer, M. B. (1991). The social self: On being the same and different at the same time. Personality and Social Psychology Bulletin, 17, 475-482. doi: 10.1177/0146167291175001

Brewer, M. B., \&Yuki, M. (2007). Culture and social identity. Em S. Kitayama, \& D. Cohen (Eds.), Handbook of cultural psychology (pp. 307-322). New York: Guilford.

Chang, M. X. L., Jetten, J., Cruwys, T., \& Haslam, C. (2017). Cultural identity and the expression of depression: A social identity perspective. Journal of Community \& Applied Social Psychology, 27(1), 16-34. doi: 10.1002/casp.2291 
Cruwys, T., South, E., Greenaway, K. H., \& Haslam, S. A. (2015). Social identity reduces depression by fostering positive attributions. Social Psychological and Personality Science, 6, 65-74. doi: 10.1177/1948550614543309

Gouveia, V. V., Athayde, R. A. A., Mendes, L. A., \& Freire, S. E. A. (2012). Introdução às medidas implícitas: Conceitos, técnicas e contribuições. Diaphora, 12, 3-16. Recuperado de http://www.sprgs.org.br/diaphora/ojs/index.php/diaphora/article/view/50/50

Gouveia, V. V., \& Clemente, M. (2000). O individualismo-coletivismo no Brasil e na Espanha: Correlatos sócio-demográficos. Estudos de Psicologia (Natal), 5, 317-346. doi: 10.1590/S1413-294X2000000200003

Gouveia, V. V., Santos, W. S., \& Milfont, T. L. (2009). O uso da estatística na avaliacão psicológica: Comentários e consideracões práticas. Em C. S. Hutz (Ed.). Avanços e polêmicas em avaliacões psicológicas (pp. 127-156). São Paulo: Casa do Psicólogo.

Greenaway, K. H., Haslam, S. A., Cruwys, T., Branscombe, N. R., Ysseldyk, R., \& Heldreth, C. (2015). From "we" to "me": Group identification enhances perceived personal control with consequences for health and well-being. Journal of Personality and Social Psychology, $109(1), 53$. doi: 10.1037/pspi0000019

Haslam, S. A. (2001). Psychology in organizations: The social identity approach. London: Sage Publications.

Hofstede, G. (1984). Culture's consequences: International differences in work-related values (Vol. 5). London: Sage Publications.

Holgado-Tello, F., Chacón-Moscoso, S., Barbero-García, I., \& Vila-Abad, E. (2010). Polychoric versus Pearson correlations in exploratory and confirmatory factor analysis of ordinal variables. Quality and Quantity, 44, 153-166. doi: 10.1007/s11135-008-9190-y

Kline, P. (2000). The handbook of psychological testing. London: Routledge.

Kline, R. B. (2011). Principles and practice of structural equation modeling (3rd ed.). New York: The Guilford Press.

Kohlsdorf, M., \& Costa, A. L., Jr. (2009). O autorrelato na pesquisa em psicologia da saúde: Desafios metodológicos. Psicologia Argumento, 27, 131-139. Recuperado de http://repositorio.unb.br/handle/10482/6028

Kreiner, G. E., \& Ashforth, B. E. (2004). Evidence toward an expanded model of organizational identification. Journal of Organizational Behavior, 25, 1-27. doi: 10.1002/job.234

Lorenzo-Seva, U., \& Ferrando, P. J. (2006). FACTOR: A computer program to fit the exploratory factor analysis model. Behavior Research Methods, 38, 88-91. doi: 10.3758/BF03192753

Lorenzo-Seva, U. (1999). Promin: A method for oblique factor rotation. Multivariate Behavioral Research, 34, 347-365. doi: 10.1207/ S15327906MBR3403_3

Lorenzo-Seva, U., Timmerman, M. E., \& Kiers, H. A. L. (2011). The Hull method for selecting the number of common factors. Multivariate Behavioral Research, 46, 340-364. doi: 10.1080/00273171.2011.564527

Markus, H. R., \& Kitayama, S. (1991). Culture and the self: Implications for cognition, emotion, and motivation. Psychological Review, 98, 224-253. doi: 10.1037/0033-295X.98.2.224

Mayhew, M. G., Gardner, J., \& Ashkanasy, N. M. (2010). Measuring individuals' need for identification: Scale development and validation. Personality and Individual Differences, 49, 356-361. doi: 10.1016/j.paid.2010.03.031

Michener, A. H., DeLamater, J. D., \& Myers, D. G. (2005). Psicologia social. São Paulo: Pioneira Thomson Leaening.

Mislevy, R. J., \& Bock, R. D. (1990). BILOG 3: Item analysis and test scoring with binary logistic regression models. Mooresville, IN: Scientific Software.

Muthén B. O., Du Toit S. H. C., \& Spisic D. (1997). Robust inference using weighted least squares and quadratic estimating equations in latent variable modeling with categorical and continuous outcomes (Manuscrito técnico não publicado). University of California, Los Angeles.

Muthén, L. K., \& Muthén, B. O. (2010). Mplus: The comprehensive modeling program for applied research (6th ed.). Los Angeles, CA: Muthén\&Muthén.

Pimentel, C. E., Gouveia, V. V., \& Fonseca, P. N. D. (2005). Escala de Identificação com Grupos Alternativos: Construção e comprovação da estrutura fatorial. Psico-USF, 10, 121-127. Recuperado de http://www.scielo.br/pdf/pusf/v10n2/v10n2a03

Pornprasertmanit, S., Miller, P., Schoemann, A., \& Rosseel, Y. (2015). Tools: Useful tools for structural equation modeling. R package version 0.4-6.

R Development Core Team. (2011). R: A language and environment for statistical computing. Vienna, Austria: R Foundation for Statistical Computing. Shapiro, A., \& Berge, J. M. F. (2002). Statistical inference of minimum rank factor analysis. Psychometrika, 67, 79-94. doi:10.1007/BF02294710

Shapiro, A., \& Ten Berge, J. M. (2002). Statistical inference of minimum rank factor analysis. Psychometrika, 67, 79-94. doi: 10.1007/BF02294710

Tabachnick, B. G., \& Fidell, L. S. (2013). Using multivariate statistics (6th ed.). Boston, MA: Allyn and Bacon.

Tajfel, H. (1978). Social categorization, social identity and social comparison. Em H. Tajfel (Ed.), Differentiation between social groups. London: Academic Press.

Tajfel, H., \& Turner, J.C. (1979). An integrative theory of intergroup conflict. Em W. Austin \& S. Worchel (Eds.), Social psychology of intergroup relations (pp. 33-47). Monterey, CA: Brooks Cole.

Tajfel, H., Flament, C., Billig, M. G., \& Bundy, R. P. (1971). Social categorization and inter-group behavior. European Journal of Social Psychology, 1, 149-177. doi: 10.1002/ejsp.2420010202

Torres, C., \& Neiva, E. R. (2011). Psicologia social. Porto Alegre, RS: Artmed.

Turner, J. C. (1982). Towards a cognitive redefinition of the social group. In H. Tajfel (Ed.), Social identity and intergroup relations (pp. 15-40). Cambridge: Cambridge University Press.

\section{Sobre os autores}

Anderson Mesquita do Nascimento é doutorando em Psicologia Social pela Universidade Federal da Paraíba e mestre em Psicologia Social pela mesma Universidade.

Valdiney Veloso Gouveia é professor titular de Psicologia Social da Universidade Federal da Paraíba e Pesquisador 1A do CNPq. É doutor em Psicologia Social pela Universidade Complutense de Madrid.

Ana Karla Silva Soares é professora da Universidade Federal do Mato Grosso do Sul. Mestre e Doutora pela Universidade Federal da Paraíba.

Jéssica Bruna Santana Silva é doutoranda em Psicologia Social pela Universidade Federal da Paraíba e mestre em Neurociência Cognitiva e Comportamento pela mesma Universidade.

Alex Sandro de Moura Grangeiro é professor da Faculdade Luciano Feijão (Sobral/Ceará). Mestre em Psicologia pela Universidade Federal do Ceará e doutor em Psicologia Social pela Universidade Federal da Paraíba. 